\title{
Method of evolving junctions: A new approach to optimal control with constraints
}

\author{
Shui-Nee Chow ${ }^{\text {a }}$, Wuchen Li $^{\text {a }}$, Jun Lu ${ }^{\text {b }}$, Haomin Zhou ${ }^{\text {a }}$

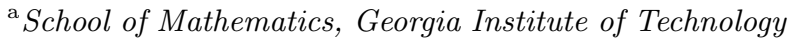 \\ ${ }^{\mathrm{b}}$ Wells Fargo Securities
}

\begin{abstract}
We propose a new strategy, called method of evolving junctions (MEJ), to compute the solutions for a class of optimal control problems with constraints on both state and control variables. Our main idea is that by leveraging the geometric structures of the optimal solutions, we recast the infinite dimensional optimal control problem into an optimization problem depending on a finite number of points, called junctions. Then, using a modified gradient flow method, whose dimension can change dynamically, we find local solutions for the optimal control problem. We also employ intermittent diffusion, a global optimization method based on stochastic differential equations, to obtain the global optimal solution. We demonstrate, via a numerical example, that MEJ can effectively solve path planning problems in dynamical environments.
\end{abstract}

Key words: optimal control; constraints; stochastic differential equations; junctions

\section{Introduction}

An optimal control problem with constraints seeks to determine the input (control) $u(t) \in \mathbb{R}^{r}$ to a dynamical system that optimizes a given performance functional (maximize profit, minimize cost, etc), while satisfying various state and/or control constraints,

$$
\min _{x, u} \int_{t_{0}}^{t_{f}} L(x(t), u(t), t) d t+\psi\left(x\left(t_{f}\right), t_{f}\right),
$$

subject to

$$
\begin{aligned}
& \dot{x}=f(x(t), u(t), t), \quad t \in\left[t_{0}, t_{f}\right], \\
& x\left(t_{0}\right)=x_{0}, \quad M\left(t_{f}, x\left(t_{f}\right)\right)=0, \\
& \phi(x(t), t) \geq 0, \quad \varphi(u(t), t) \geq 0, \quad t \in\left[t_{0}, t_{f}\right],
\end{aligned}
$$

where the state variable $x(t) \in \mathbb{R}^{n}$ is often called the trajectory or path in the phase space. $u(t) \in \mathbb{R}^{r}$ is the

\footnotetext{
* This paper was not presented at any IFAC meeting. Corresponding author Wuchen Li. Tel. 678-907-5128. This work is partially supported by NSF Awards DMS-1419027, DMS-1620345, and ONR Award N000141310408.

Email addresses: chow@math.gatech.edu (Shui-Nee Chow), wli83@gatech.edu (Wuchen Li), junlu0@icloud.com (Jun Lu), hmzhou@math.gatech.edu (Haomin Zhou).
}

Preprint submitted to Automatica control variable; $L: \mathbb{R}^{n} \times \mathbb{R}^{r} \times \mathbb{R}^{+} \rightarrow \mathbb{R}$ is the running cost; $\psi: \mathbb{R}^{n} \times \mathbb{R}^{+} \rightarrow \mathbb{R}$ is the terminal cost, and $t_{f}$ is the terminal time, which may be undetermined in some problems. $\phi: \mathbb{R}^{n} \times \mathbb{R}^{+} \rightarrow \mathbb{R}^{p}$ is the state constraint and $\varphi: \mathbb{R}^{r} \times \mathbb{R}^{+} \rightarrow \mathbb{R}^{q}$ is the control constraint. For technical simplicity, we assume that $L, \phi, \varphi, M$ are continuously differentiable with respect to $x$ and $t$ in this paper.

Because many engineering problems can be formulated into the framework of (1) [23,32], optimal control theory has vast applications $[2,3,20]$. However, due to the complexity of those applications, few of them can be solved analytically. Thus numerical methods are often employed instead. Traditionally, the methods are divided into three categories, (1) state-space, (2) indirect, and (3) direct methods. State-space approaches apply the principle of dynamic programming, which states that each sub-arc of the optimal trajectory must be optimal. It leads to the well-known Hamilton-JacobiBellman (HJB) equations, which are non-linear partial differential equations (PDEs) [5,35]. Indirect methods employ the necessary condition of optimality known as Pontryagin's maximum Principle [37]. This leads to a boundary value problem, which is then solved by numerical methods. Thus this approach is also referred to as "first optimize, then discretize". Typical examples are neighboring extremal algorithm, gradient algorithm, and quasi-linearization algorithm [4,8,27,33], just 
to name a few. Direct methods take the idea of "first discretize, then optimize". They convert the original continuous infinite dimensional control problem into a finite dimensional optimization problem. The resulting discrete problem becomes a large scale standard nonlinear programming problem (NLP) which can be solved by many well established algorithms such as Newton's method, Quasi-Newton methods $[1,14,15,17,18,25,28-30,36]$. Direct methods are nowadays the most widespread and successfully used techniques.

Different from the existing methods, in this paper, we design a new fast numerical method focusing on a class of optimal control problems called separable problems [10-12,31]. Simply put, a path is said to be separable, if there exists finite number of points, called junctions, that divide the path into segments, such that a constraint can only switch from inactive to active (or vice versa) at junctions.

The significance of being separable is that the determination of the entire path boils down to the determination of only a finite number of junctions and the determination of a finite number of optimal trajectories of smaller sizes, for which the constraints are either inactive or active on the entire segment. On the other hand, in many applications, the optimal solution on each segment can be computed either analytically or numerically by efficient algorithms. In this way, the original infinite dimensional problem of finding the whole path is converted into a finite dimensional problem, i.e. determining a finite number of junctions. Thus one may ${ }^{1}$ gain a tremendous dimensional reduction.

The resulting finite dimensional problem can be handled by many established algorithms, for example, the gradient descent method. In this case, each steady state of the gradient descent flow can be a local minimizer. It is evident from many applications that the total number of minimizers can often be very large. Therefore, it is highly desirable to design methods that are capable of obtaining the global optimal trajectory. In this paper, we adopt a recently developed global optimization strategy, called intermittent diffusion (ID) [13]. The idea is to add noises (diffusions) to the gradient flow intermittently. When the noise is turned off, one gets a pure gradient flow and it quickly converges to a local minimizer. When the noise is turned on, the perturbed flow becomes stochastic differential equations (SDEs), which has a positive probability to jump out of local traps and converges to other minimizers, including the global one. It can be shown that the local minimizers obtained will include the global one with probability arbitrarily close to 1 if appropriate perturbations are added. We call the method outlined above Method of Evolving Junctions (MEJ).

\footnotetext{
1 We assume that constraints can be handled efficiently through functions of junctions.
}

In the literature, the concept of a junction has been introduced in the past $[19,22]$, and used in indirect methods $[6,7]$. Most of them use junctions as shooting parameters to solve the Hamiltonian systems. For example, the one proposed in [6] uses a continuation method, also called homotopy method, together with the shooting method for the boundary value ODEs derived from the maximum principle. This is different from how we use junctions. Namely, we directly derive equations that govern the evolution of junctions to achieve the optimal control requirements.

Because MEJ is designed for separable problems, especially for those whose optimal solutions and constraints can be dealt efficiently through junctions, it has the ability to overcome some well-known limitations of the aforementioned three methods. Namely, the HJB approach, which gives the global solution, can be computationally expensive and suffers from the notorious problem known as "curse of dimensionality". Indirect methods guarantee to find local optimal solutions, while finding the global optimal solutions, if possible, requires carefully designed initializations. Direct methods often require finer discretization (smaller time steps) if better accuracy is desired, which increases computational demands. In recent years, various efforts have been devoted to reduce the computational cost [28-30].

We arrange this paper as follows. In Section 2, we explain the idea of separability and give the algorithm for MEJ. In Section 3, we use the new method to solve a robotic path-planning problem, through which we demonstrate the advantages of MEJ.

\section{Method of Evolving Junctions}

To simplify our discussion, we introduce MEJ to (1) with only state constraints. Its extension to control constraints is straightforward and hence omitted here.

\subsection{The Separable Structure}

Definition 1 A path $x(t)$ is said to be separable if there exists a finite partition: $t_{0}<t_{1}<t_{2}<\cdots<t_{N}<$ $t_{N+1}=t_{f}$ such that $\left.x(t)\right|_{\left[t_{i}, t_{i+1}\right]}$ alternates between segments where constraints are either active or inactive. An optimal control problem (1) is called separable if its optimal path is separable.

The notion of separability has been previously described in [38] where only trajectories consisting of three parts are considered $(N=2)$. Here we define $\tilde{x}_{i}:=\left(t_{i}, x\left(t_{i}\right)\right)$ and call them junctions. A junction pair $\tilde{x}_{i}, \tilde{x}_{i+1}$ determines a trajectory connecting them, which solves either the optimal control in the free space or with active constraints, denoted by $\gamma_{0}\left(\tilde{x}_{i}, \tilde{x}_{i+1}\right)$ or $\gamma_{c}\left(\tilde{x}_{i}, \tilde{x}_{i+1}\right)$ respectively. 
The separability allows us to restrict the search of optimal trajectories in a subset $H$ defined by

$$
H:=\{\gamma: \gamma \text { is determined by finite junctions }\}
$$

More precisely, if $\gamma \in H$, there exists a sequence of junctions on the boundary of the constraints, $\left(\tilde{x}_{0}, \cdots, \tilde{x}_{N}, \tilde{x}_{N+1}\right)$ such that $\gamma$ can be represented as

$$
\gamma_{1}\left(\tilde{x}_{0}, \tilde{x}_{1}\right) \cdot \gamma_{2}\left(\tilde{x}_{1}, \tilde{x}_{2}\right) \cdots \gamma_{N}\left(\tilde{x}_{N}, \tilde{x}_{N+1}\right)
$$

where $\gamma_{i}$ is either $\gamma_{0}$ or $\gamma_{c}$, and $\gamma_{i} \cdot \gamma_{i+1}$ denotes the concatenation of two trajectories.

As a result, the cost functional of (1) can be expressed as a function of junctions:

$$
J\left(\tilde{x}_{1}, \cdots, \tilde{x}_{N+1}\right):=\sum_{i=1}^{N} J_{i}\left(\tilde{x}_{i}, \tilde{x}_{i+1}\right)
$$

where $J_{i}$ is the cost on $\gamma_{i}$, i.e.

$$
J_{i}\left(\tilde{x}_{i}, \tilde{x}_{i+1}\right):=\min _{x, u} \int_{t_{i}}^{t_{i+1}} L(x(t), u(t), t) d t
$$

Moreover, the optimal trajectory connecting $\tilde{x}_{i}$ and $\tilde{x}_{i+1}$ must not violate the constraints,

$$
V\left(\tilde{x}_{i}, \tilde{x}_{i+1}\right):=\min _{t_{i} \leq t \leq t_{i+1}, 1 \leq k \leq p} \phi_{k}\left(\gamma_{i}(t), t\right)=0 .
$$

For convenience, we call $V\left(\tilde{x}_{i}, \tilde{x}_{i+1}\right)=0$ the visibility constraints.

For problems with separable structures, any optimal trajectories must be in $H$. Therefore, in order to find an optimal trajectory, only the optimal junctions need to be computed. In other words, problem (1) can be recast as

$$
\min _{\tilde{x}_{1}, \cdots, \tilde{x}_{N+1}} J\left(\tilde{x}_{1}, \cdots, \tilde{x}_{N+1}\right)
$$

subject to $V\left(\tilde{x}_{i}, \tilde{x}_{i+1}\right)=0$.

We must point out that to evaluate $J_{i}\left(\tilde{x}_{i}, \tilde{x}_{i+1}\right)$, we need to be able to find $\gamma_{i}$ efficiently. This requires us to solve (1) with $\tilde{x}_{i}$ and $\tilde{x}_{i+1}$ being two ending conditions, while no constraint changes from inactive to active, or vice versa, between two junctions. In addition to solving $\gamma_{i}$, we need to calculate the constraint function $V$ efficiently as well. If these can be achieved by either analytical solutions or rapid numerical methods, we convert (1) into a finite dimensional optimization problem. Such problems include, for example, many linear quadratic optimal control problems, for which analytical solutions are available, and path-planning related problems as we will illustrate in this paper.
We emphasize that solving (4) must face two challenges. One is how to find the global minimizer. The other is that the dimension of the optimization problem is unknown, since the number and index of junctions can not be determined a priori. We apply intermittent diffusion (ID) to conquer the first problem, and a new procedure of inserting and removing junctions from the system to treat the second challenge. They are presented in the next two subsections.

\subsection{Intermittent Diffusion}

ID is a global optimization strategy developed in [13]. For our problems, it involves finding the global minimizers of (4) by SDEs on the manifolds defined by the boundaries of the constraints,

$$
d \tilde{x}=P_{\tilde{x}}[-\nabla J(\tilde{x}) d \theta+\sigma(\theta) d W(\theta)],
$$

where $\tilde{x}=\left(\tilde{x}_{0}, \tilde{x}_{1}, \cdots, \tilde{x}_{N}, \tilde{x}_{N+1}\right), \theta$ is an artificial time variable that is different from $t, W(\theta)$ the standard Brownian motion, and $P_{\tilde{x}}$ the orthogonal projection onto the tangent space at $\tilde{x}$. If we denote the set of feasible directions at $\tilde{x}$ as

$$
\mathcal{F}(\tilde{x})=\left\{\mathbf{q} \mid \nabla V\left(\tilde{x}_{i}, \tilde{x}_{i+1}\right) \cdot \mathbf{q}=0,\|\mathbf{q}\|=1\right\}
$$

then $P_{\tilde{x}}(\mathbf{p})$ is defined by

$-\frac{P_{\tilde{x}}(\mathbf{p})}{\left\|P_{\tilde{x}}(\mathbf{p})\right\|}=\arg \min _{\mathbf{q} \in \mathcal{F}(\tilde{x})} \mathbf{q} \cdot \mathbf{p}, \quad\left\|P_{\tilde{x}}(\mathbf{p})\right\|=\min _{\mathbf{q} \in \mathcal{F}(\tilde{x})}|\mathbf{q} \cdot \mathbf{p}|$.

For convenience, we denote $\nabla^{c} J(\tilde{x}):=P_{\tilde{x}}(\nabla J(\tilde{x}))$.

When giving the definition for $\mathcal{F}(\tilde{x})$, we assume that the visibility function $V$ is differentiable with respect to the junctions. This is true for many problems we consider. For instance, the example we present in this paper has a smooth analytical formula for $V$. However, the differentiability for general $V$ s may not be determined easily. A different definition for $P_{\tilde{x}}$ must be introduced to accommodate the challenge of non-differentiable $V$ s.

The function $\sigma(\theta)$ is piecewise constant, controlling the amount of noise added intermittently. More precisely, $\sigma(\theta)=\sum_{j=1}^{m} \sigma_{j} \chi_{\left[S_{j}, T_{j}\right]}(\theta)$, where $\left\{\left[S_{j}, T_{j}\right]\right\}_{j=1}^{m}$ are disjoint intervals, and $\chi_{\left[S_{j}, T_{j}\right]}$ is the characteristic function on $\left[S_{j}, T_{j}\right]$. If $\sigma(\theta) \neq 0,(5)$ is a well-defined SDE [21], whose solution has a positive probability to escape the attraction of a minimizer. If $\sigma(\theta)=0$, we obtain the projected gradient flow, whose solution converges to a minimizer. The theory of ID suggests that the solutions of the SDEs visit the global minimizers with probability arbitrarily close to 1 if the computation time (intermittent diffusion step) is long enough. In principle, the more number of intermittent intervals we use (more expensive in computation), the larger the probability of achieving the global optimal solution. Details can be found in [13]. 


\subsection{Handling Dimension Changes}

To maintain separability, we introduce the following two operations to add or remove junctions as needed.

Insert junctions. During the evolution of junctions according to $(5), \gamma_{k}\left(\tilde{x}_{k}, \tilde{x}_{k+1}\right)$ may intersect with the interior of the constrained region. The new junctions are added through a set

$E_{k}=\left\{(t, \gamma(t)) \mid \min _{1 \leq s \leq p} \phi_{s}\left(\gamma_{k}(t), t\right)=0, \quad t \in\left(t_{k}, t_{k+1}\right)\right\}$

To illustrate the idea, we assume $\gamma_{k}\left(\tilde{x}_{k}, \tilde{x}_{k+1}\right)$ intersects with the constraint at $\tilde{y}$ for the first time ${ }^{2}$ as shown in Fig 1 . We have $E_{k}=\{\tilde{y}\}$. So we add $\tilde{y}$ as a new junction and the path is determined by $\left(\tilde{x}_{k}, \tilde{y}, \tilde{y}, \tilde{x}_{k+1}\right)$. With the new set of junctions, we have another gradient flow which is also expressed by (5). However, the number of equations is larger.

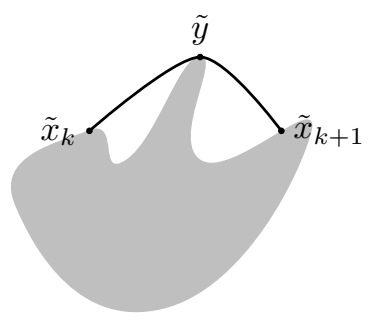

Fig. 1. New junctions $\tilde{y}$ is added if $\gamma_{k}\left(\tilde{x}_{k}, \tilde{x}_{k+1}\right)$ intersects with the boundary of the constraints (grey area).

A general algorithm for the insertion procedure is given here.

\section{Inserting algorithm:}

Input: Junctions $\tilde{x}=\left(\tilde{x}_{0}, \cdots, \tilde{x}_{N+1}\right)$.

1. for $k=0: N$;

2. Find the optimal path $\gamma_{k}(t)$ connecting $\tilde{x}_{k}, \tilde{x}_{k+1}$;

3. Compute $^{3}$

$$
a=\operatorname{sign}\left\{\min _{1 \leq s \leq p, t_{i} \leq k \leq t_{i+1}} \phi_{s}\left(\gamma_{k}(t), t\right)\right\} ;
$$

4. If $a<0$;

5. $\quad$ Find all $t^{*} \in\left(t_{k}, t_{k+1}\right)$, such that

$$
\min _{1 \leq s \leq p} \phi_{s}\left(\gamma_{k}\left(t^{*}\right), t^{*}\right)=0
$$

2 In general, there may exist multiple intersection points. We need to find all junction points defined in $E_{k}$.

3 Depending on the problem (1), one may solve $a$ by either an analytical formula or numerical methods (line search or Newton's method).
6. $\quad$ Denote the new junctions $\tilde{x}^{*}=\left(t^{*}, \gamma_{k}\left(t^{*}\right)\right)$;

7. Update junctions:

$$
\tilde{x} \rightarrow\left\{\tilde{x}_{0}, \cdots, \tilde{x}_{k-1}, \tilde{x}^{*}, \tilde{x}^{*}, \tilde{x}_{k+1}, \cdots, \tilde{x}_{N+1}\right\}
$$

8. end

9. end

Remove junctions. Junctions need to be removed if doing so results in a path with smaller cost. For example, as depicted in Fig 2, when two junctions $\tilde{x}_{k}$ and $\tilde{x}_{k+1}$ on the boundary meet each other during the flow, i.e. $\tilde{x}_{k}=\tilde{x}_{k+1}$, we remove them from the junction list. The triangle inequality,

$$
J_{0}\left(\tilde{x}_{k-1}, \tilde{x}_{k+2}\right) \leq J_{0}\left(\tilde{x}_{k-1}, \tilde{x}_{k}\right)+J_{0}\left(\tilde{x}_{k}, \tilde{x}_{k+2}\right),
$$

indicates that the original path $\left(\cdots, \tilde{x}_{k-1}, \tilde{x}_{k}, \tilde{x}_{k+1}, \tilde{x}_{k+2}, \cdots\right)$ has cost no less than the path $\left(\cdots, \tilde{x}_{k-1}, \tilde{x}_{k+2}, \cdots\right)$. However, the new segment $\gamma_{k-1}\left(\tilde{x}_{k-1}, \tilde{x}_{k+2}\right)$ may intersect with the boundary of constraints. Hence, we may add the new intersections into the set of junctions as needed.

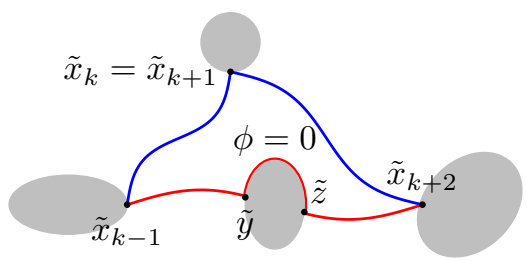

Fig. 2. Remove junctions when $\tilde{x}_{k}=\tilde{x}_{k+1}$. The blue is the original path and the red is the new path.

We summarize above steps into the following junction removal algorithm:

\section{Removing algorithm:}

Input: Junctions $\tilde{x}=\left(\tilde{x}_{0}, \cdots, \tilde{x}_{N+1}\right)$. Tolerance $\epsilon$. 1. for $k=0: N$;

2. If $\left\|\tilde{x}_{k}-\tilde{x}_{k+1}\right\| \leq \epsilon$;

3. $\quad$ Remove the junction pair $\tilde{x}_{k}, \tilde{x}_{k+1}$ from $\tilde{x}$;

4. Compute the optimal path $\gamma_{k-1}(t)$ between a new pair $\tilde{x}_{k-1}, \tilde{x}_{k+2}$;

5. $\quad$ Apply Inserting algorithm for $\gamma_{k-1}(t)$;

6. end

7. end

\subsection{Algorithm}

Now we are ready to state our algorithm.

\section{Method of Evolving Junctions \\ Input: Constraint $\phi$ and $\psi$; starting and ending points $x_{0}$ and $M$; running cost $L$, terminal time $t_{f}$, and ODE $f$;}


number of intermittent diffusion intervals $m$.

Output: The optimal set $\gamma_{o p t}$ of junctions.

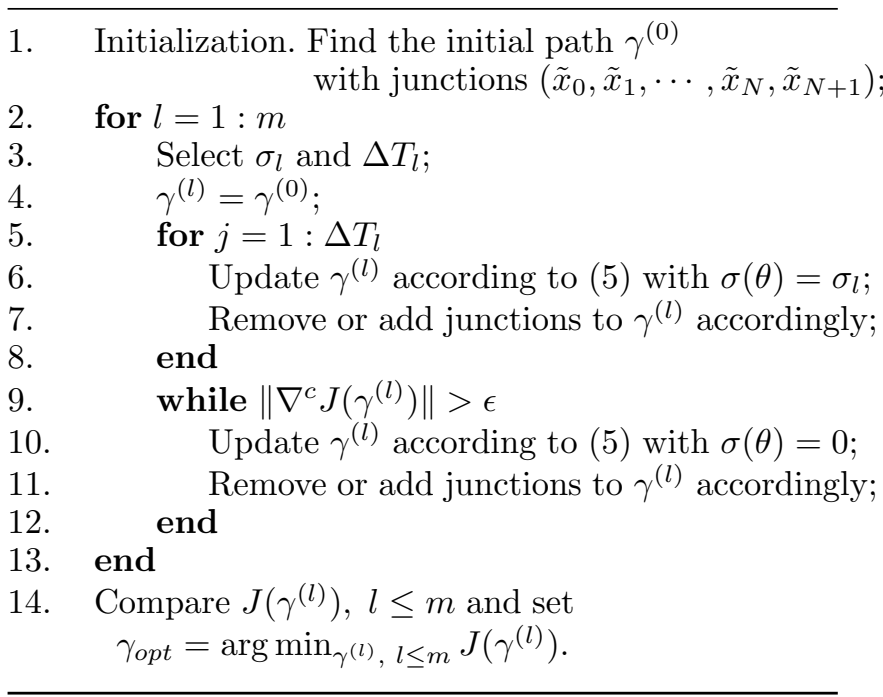

In practice, we find the inital path and junctions by solving (1) without constraints, in which the solution is an initial path $\gamma^{(0)}$. Then, we find all intersections of $\gamma^{(0)}$ with the boundaries of constraints, which are the initialization of junctions.

Remark 1 For the computation of $\nabla^{c} J(\tilde{x})$ in step 6 and 10 , some simple formulas can be used in term of (1)'s costates, see [34] for more details.

\section{Numerical experiment}

In this section, we present an example solved by MEJ. More examples can be found in [24], in which we also compare MEJ for some standard linear quadratic optimal control problems with methods reported in $[26,40]$.

Example: Optimal path with moving obstacles. This example is motivated by practical problems in robotics, in which the problem is considered as very challenging on its own $[16,39]$.

In simple words, our goal is to find the optimal path $\gamma(t) \in \mathbb{R}^{2}$ for a robot moving from a starting point $X$ to a target location $Y$ with minimal cost, while avoiding collisions with several polygonal obstacles that also move in the environment. To be mathematically precise, we want to solve the optimal control problem:

$$
\min _{\gamma, v} \int_{0}^{T} L(\gamma(t), v(t), t) d t
$$

subject to

$$
\begin{aligned}
& \dot{\gamma}(t)=v(t), \quad t \in[0, T] ; \quad \gamma(0)=X, \quad \gamma(T)=Y ; \\
& \gamma(t) \in \mathbb{R}_{c}^{2}(t), \quad\|v(t)\| \leq v_{m},
\end{aligned}
$$

where $v$ and $t$ represent the velocity and time respectively, the constant $v_{m}$ is the maximal speed that the robot can move, $\|\cdot\|$ is the 2 -norm, and $\mathbb{R}_{c}^{2}(t)$ is the time dependent obstacle-free space defined as follows: Let $P_{1}(t), \cdots, P_{K}(t)$ be $K$ time-dependent open subsets of $\mathbb{R}^{2}$ representing the moving obstacles, and $\mathbb{R}_{c}^{2}(t)=$ $\mathbb{R}^{2} \backslash\left(\cup_{i=1}^{K} P_{i}(t)\right)$.

In particular, we consider the moving environment of a 2 -D square region $[0,2] \times[0,2]$ containing 5 moving polygons with different constant velocities. The running cost is chosen to be a monotonically increasing convex function

$$
L(\gamma, v, t)=\|v\|^{2}+c .
$$

For convenience, we pick the starting point as $(0,1)$, and the ending point $(2,1)$ with maximal speed constraint $v_{m}=10$ and $c=10$. The terminal time $T$ is unknown.

Denote the $k$ th obstacle by $P_{k}$, and assume that $\alpha_{k}(x):[0,2 \pi] \rightarrow \mathbb{R}^{2}$ is the normalized arc-length parametrization of its boundary. If $P_{k}$ moves with a constant velocity $\mathbf{v}_{k}$, then its moving boundary can be parametrized by $R_{k}(x, t)=\alpha_{k}(x)+\mathbf{v}_{k} t$. Hence a junction $\tilde{x}_{i}=\left(t_{i}, x_{i}\right)$ describes a postion $R_{k}\left(\tilde{x}_{i}\right)=R_{k}\left(x_{i}, t_{i}\right) \in \mathbb{R}^{2}$.

Step 1: We solve the optimal path in the free space. We want to compute the optimal solution for two junctions $\tilde{x}_{i}=\left(t_{i}, x_{i}\right), \tilde{x}_{i+1}=\left(t_{i+1}, x_{i+1}\right)$ on two different polygons. For convenience, we name them $P_{1}, P_{2}$. See Figure 3 .

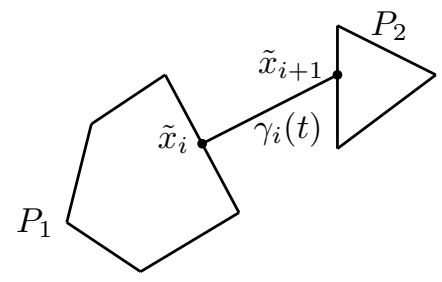

Fig. 3. Junctions on different polygons.

The optimal path satisfies the Euler-Lagrange equation

$\nabla_{\gamma} L(\gamma, v, t)-\frac{d}{d t} \nabla_{v} L(\gamma, v, t)=0 \Rightarrow-2 \frac{d}{d t}(\dot{\gamma})=2 \ddot{\gamma}=0$

which implies that the optimal trajectory has zero acceleration. Hence

$$
\gamma_{i}(t)=\frac{R_{2}\left(\tilde{x}_{i+1}\right)-R_{1}\left(\tilde{x}_{i}\right)}{t_{i+1}-t_{i}}\left(t-t_{i}\right)+R_{1}\left(\tilde{x}_{i}\right)
$$

and

$$
J_{i}\left(\tilde{x}_{i}, \tilde{x}_{i+1}\right)=\frac{\left(R_{2}\left(\tilde{x}_{i+1}\right)-R_{1}\left(\tilde{x}_{i}\right)\right)^{2}}{t_{i+1}-t_{i}}+c\left(t_{i+1}-t_{i}\right) .
$$


Step 2: We solve the optimal control with active constraints. There are three cases: 1 ) both state and control constraints are active; 2 ) the control constraint is active while the state constraint is not; and 3) the state constraint is active but the control constraint is not. Cases 1) and 2) can be solved easily, because the optimal path is uniquely determined by the speed constraint, which is the straight line motion between two junctions with maximal speed.

Hence we focus on 3). We want to compute the optimal solution connecting two junctions $\tilde{x}_{i}, \tilde{x}_{i+1}$ on one polygon. Without causing confusion, we also call it $P_{1}$. We have a new optimal control problem:

$$
\min _{\gamma} \int_{t_{i}}^{t_{i+1}}\left(\dot{\gamma}^{2}+c\right) d t
$$

subject to only state constraints $\gamma(t)=\alpha_{1}(x(t))+\mathbf{v}_{1} t$, $x\left(t_{i}\right)=x_{i}$ and $x\left(t_{i+1}\right)=x_{i+1}$. We can rewrite the running cost in term of a new variable $x(t)$ :

$$
L_{1}(x, \dot{x}, t)=\dot{x}^{2}(t)+\alpha_{1 x}(x(t)) \cdot \mathbf{v}_{1} \dot{x}(t)+\left\|\mathbf{v}_{1}\right\|^{2}+c .
$$

Since $P_{1}$ is a polygon, $\alpha_{1 x}(x)$ is a piecewise constant function. So we need to solve (8) for two scenarios, according to whether $x_{i}$ and $x_{i+1}$ are on the same edge or not. See Figure 4 (a) and (b).

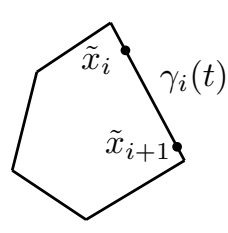

(a)

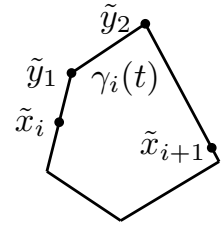

(b)
Fig. 4. Junctions on a polygon.

Scenario (a), junctions $\tilde{x}_{i}, \tilde{x}_{i+1}$ are on the same edge of $P_{1}$. Here $\alpha_{1 x}(x)$ is a constant vector and $\beta=\alpha_{1 x}(x(t))$. $\mathbf{v}_{1}$ is just a constant. Then (8) becomes

$$
\min _{x} \int_{t_{i}}^{t_{i+1}}\left(\dot{x}(t)^{2}+\beta \dot{x}(t)+\left\|\mathbf{v}_{1}\right\|^{2}+c\right) d t,
$$

subject to $x\left(t_{i}\right)=x_{i}, x\left(t_{i+1}\right)=x_{i+1}$. This is an optimal control problem without constraint. By the EulerLagrange equation, the optimal solution satisfies

$$
\nabla_{x} L_{1}(x, \dot{x}, t)-\frac{d}{d t} \nabla_{\dot{x}} L_{1}(x, \dot{x}, t)=0 \Rightarrow \ddot{x}(t)=0 .
$$

Hence the optimal path moves with a relative constant velocity:

$$
\gamma_{i}(t)=\frac{R_{1}\left(\tilde{x}_{i+1}\right)-R_{1}\left(\tilde{x}_{i}\right)}{t_{i+1}-t_{i}}\left(t-t_{i}\right)+R_{1}\left(\tilde{x}_{i}\right)
$$

and its cost is

$$
J_{i}\left(\tilde{x}_{i}, \tilde{x}_{i+1}\right)=\frac{\left(R_{1}\left(\tilde{x}_{i+1}\right)-R_{1}\left(\tilde{x}_{i}\right)\right)^{2}}{t_{i+1}-t_{i}}+c\left(t_{i+1}-t_{i}\right) .
$$

Scenario (b), junctions $\tilde{x}_{i}, \tilde{x}_{i+1}$ are on two different edges of $P_{1}$. Without loss of generality, let us assume that $x(t)$ travels clockwise on the edges of $P_{1}$, passing through nodes $l_{1}, l_{2}, \cdots, l_{m}$ of $P_{1}$ along its way. Denote $\tau_{i}$ the time when $x(t)$ reaches node $l_{i}$, i.e. $x\left(\tau_{i}\right)=l_{i}$. Then we may treat $\tilde{y}_{k}=\left(\tau_{k}, l_{k}\right)$ as a new junction, with only $\tau_{k}$ to be determined, see Figure 4 (b) for an illustration. Furthermore, we can split (8) as

$$
\begin{aligned}
J_{i}^{+} & :=\min _{x}\left(\int_{t_{i}}^{\tau_{1}}+\sum_{k=1}^{m-1} \int_{\tau_{k}}^{\tau_{k+1}}+\int_{\tau_{m}}^{t_{i+1}}\right) L_{1}(x, \dot{x}, t) d t \\
& =\min _{\tilde{y}_{1}, \cdots, \tilde{y}_{m}} J\left(\tilde{x}_{i}, \tilde{y}_{1}\right)+\sum_{k=1}^{m-1} J\left(\tilde{y}_{k}, \tilde{y}_{k+1}\right)+J\left(\tilde{y}_{m}, \tilde{x}_{i+1}\right) .
\end{aligned}
$$

We note that $\tilde{y}_{k}$ and $\tilde{y}_{k+1}$ are on the same edge, its cost $J\left(\tilde{y}_{k}, \tilde{y}_{k+1}\right)$, following the solution formula (9) for Scenario (a), can be easily expressed as

$$
J\left(\tilde{y}_{k}, \tilde{y}_{k+1}\right)=\frac{\left|e_{k}\right|^{2}}{\Delta \tau_{k}}+c \Delta \tau_{k}
$$

where $\left|e_{k}\right|$ is the arc-length of the edge $e_{k}$ between nodes $l_{k}$ and $l_{k+1}$, and $\Delta \tau_{k}=\left(\tau_{k+1}-\tau_{k}\right)$. Therefore, (10) becomes

$\min _{\Delta \tau_{0}, \cdots, \Delta \tau_{m}} \frac{\left|x_{i}-l_{1}\right|^{2}}{\Delta \tau_{0}}+\sum_{k=1}^{m-1} \frac{\left|e_{k}\right|^{2}}{\Delta \tau_{k}}+\frac{\left|x_{i+1}-l_{m}\right|^{2}}{\Delta \tau_{m}}+c\left(t_{i+1}-t_{i}\right)$,

subject to $\sum_{k=0}^{m} \Delta \tau_{k}=t_{i+1}-t_{i}, \Delta \tau_{k}>0$. Here $\Delta \tau_{0}=$ $\tau_{1}-t_{i}$ and $\Delta \tau_{m}=t_{i+1}-\tau_{m}$.

In fact, (11) is a convex optimization with a linear constraint, whose solution can be obtained explicitly via solving the standard Karush-Kuhn-Tucker condition. The resulting minimal cost has an analytical expression

$$
J_{i}^{+}=\frac{\left(\left|x_{i}-l_{1}\right|+\left|l_{m}-x_{i+1}\right|+\sum_{k=1}^{m-1}\left|e_{k}\right|\right)^{2}}{t_{i+1}-t_{i}}+c\left(t_{i+1}-t_{i}\right) .
$$

In a similar manner, we can obtain the optimal cost $J_{i}^{-}$ for the path moving counterclockwise along the boundary of $P_{1}$ from $\tilde{x}_{i}$ to $\tilde{x}_{i+1}$. Hence, we get

$$
J_{i}\left(\tilde{x}_{i}, \tilde{x}_{i+1}\right)=\min \left\{J_{i}^{+}, J_{i}^{-}\right\}
$$

Step 3: We describe the constraints in terms of junctions through visibility functions. 
First, we consider the constraint that a feasible path must be outside of the obstacles, i.e. given two junctions $\tilde{x}_{i}$ and $\tilde{x}_{i+1}$, the optimal path $\gamma_{i}(t)$ connecting them should be outside of any polygon $P$. The path $\gamma_{i}(t)$ does not intersect a moving edge $e_{k}(t)$ of $P$ if $\max _{k} d_{e_{k}(t)}\left(\gamma_{i}(t)\right)>0$, where $d_{e_{k}(t)}(\gamma(t))$ is the signed distance from $\gamma(t)$ to $e_{k}(t)$. Thus the visibility function becomes

$$
V\left(\tilde{x}_{i}, \tilde{x}_{i+1}\right)=\min _{t_{i} \leq t \leq t_{i+1}} \max _{k} d_{e_{k}(t)}\left(\gamma_{i}(t)\right)=0 .
$$

The control constraints between junctions $\tilde{x}_{i}$ and $\tilde{x}_{i+1}$ can also be simply determined by,

$$
U\left(\tilde{x}_{i}, \tilde{x}_{i+1}\right)=\max _{t_{i} \leq t \leq t_{i+1}}\|\dot{\gamma}(t)\| \leq v_{m}
$$

Combining all steps, the optimal control problem (7) is transformed into the following finite dimensional optimization problem:

$$
\min _{\tilde{x}_{1}, \ldots, \tilde{x}_{N+1}} \sum_{i=1}^{N} J_{i}\left(\tilde{x}_{i}, \tilde{x}_{i+1}\right)
$$

s.t. $V\left(\tilde{x}_{i}, \tilde{x}_{i+1}\right)=0$ and $U\left(\tilde{x}_{i}, \tilde{x}_{i+1}\right) \leq v_{m}$. And this is the problem we compute using MEJ.

The initial junctions are chosen as the intersection time and positions of a straight line trajectory with a constant speed connecting the initial and end points. The procedure of inserting junctions requires determining whether a constant moving line intersects with a polygonal, which can be solved by using either line search or Newton's method. MEJ finds two minimizers. The global one has cost 5.844533, while the local one has cost 5.846041. We depict snapshots of the two paths in Figures 5 and 6 respectively. The global minimizer has 8 junctions while the local one only has 4 .

\section{Conclusion}

In summary, we present MEJ for the separable optimal control problems with both state and control constraints. When applying it to certain separable problems, the method may have following advantages compared to existing methods:

(1) Significant dimensional reduction. Optimal control problems are in general considered as infinite dimensional problems in Banach spaces. By leveraging the separability structure of the optimal solution, MEJ reformulates the objectives and constraints in terms of junctions living on the boundaries of the constraints. This may fundamentally change the computation complexity and achieve

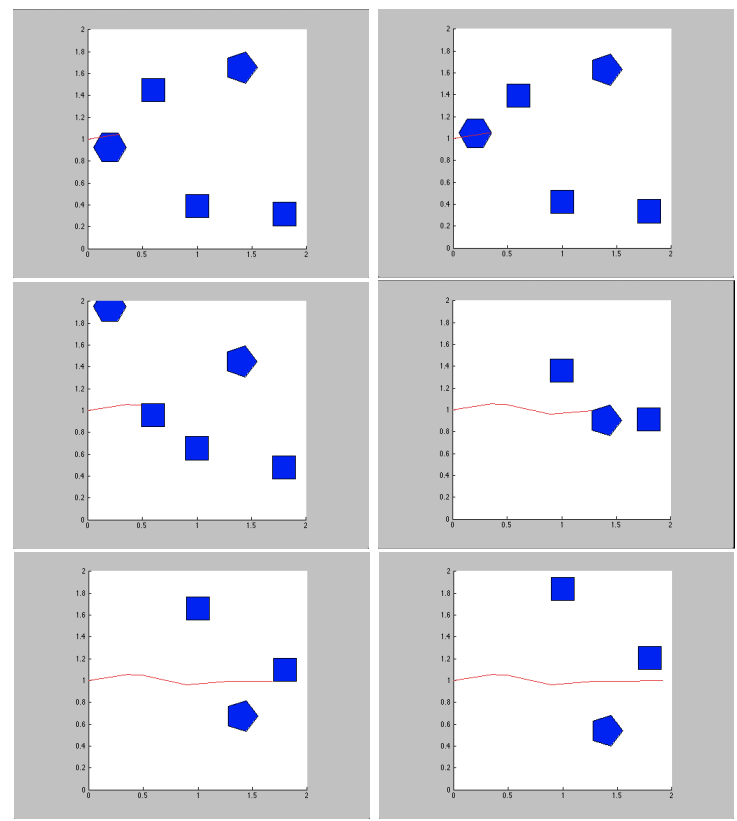

Fig. 5. Snapshots of the global minimizer.

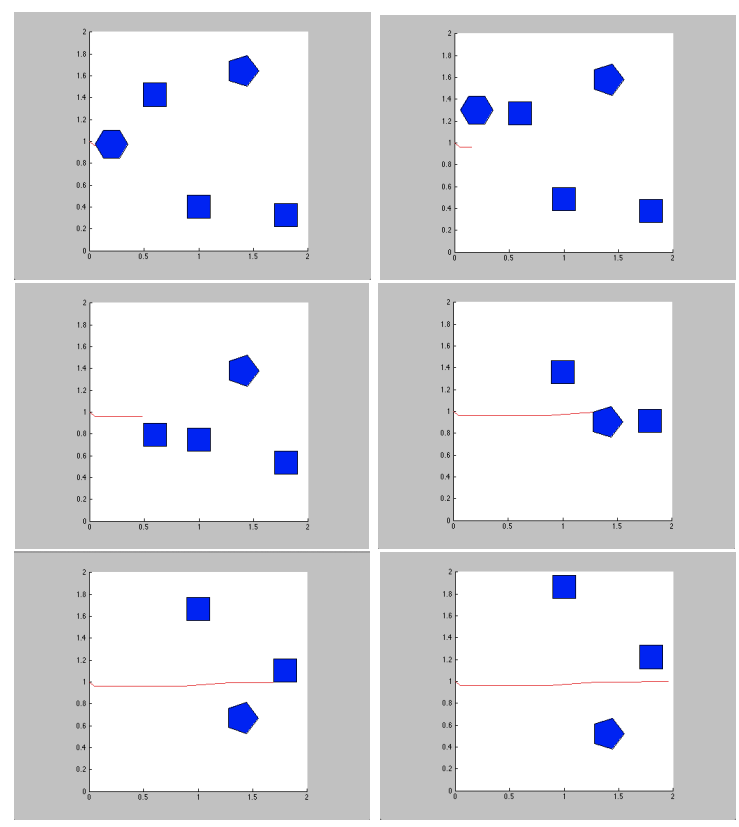

Fig. 6. Snapshots of the local minimizer.

significant dimensional reduction for some problems.

(2) Fast and accurate. MEJ only needs to solve initial value SDEs. So it has the potential to be very fast. Our experiments confirm this claim.

(3) MEJ has the potential to find the globally optimal trajectory as well as a series of locally optimal trajectories through the adoption of ID.

On the other hand, MEJ creates some theoretical questions that are very interesting on their own. For exam- 
ple, the SDEs solved by MEJ may change dimensions dynamically during its course, and the time and location of the change cannot be prescribed a priori. This is a question that has not been studied in mathematics at all. Comparing to the existing methods for optimal control problems, MEJ requires customized reformulation to convert the original problem to a constrained optimization in terms of junctions. For certain problems, this may not be a trivial tasks. Those are among the questions that we are investigating in our study.

Acknowledgement: The authors would like to thank Professor Magnus Egerstedt for many fruitful and inspirational discussions on the related topics.

\section{References}

[1] Behcet Acikmese and Lars Blackmore. Lossless convexification of a class of optimal control problems with non-convex control constraints. Automatica, 47(2):341-347, 2011.

[2] Fabio Ancona and Alberto Bressan. Patchy vector fields and asymptotic stabilization. ESAIM: Control, Optimisation and Calculus of Variations, 4:445-471, 1999.

[3] Fabio Ancona and Alberto Bressan. Nearly time optimal stabilizing patchy feedbacks. In Annales de l'Institut Henri Poincare (C) Non Linear Analysis, volume 24, pages 279310. Elsevier, 2007.

[4] Alampallam V Balakrishnan and Lucien W Neustadt. Computing methods in optimization problems: proceedings. Academic Press, 1964.

[5] Richard Bellman. Dynamic programming and the smoothing problem. Management Science, 3(1):111-113, 1956.

[6] Joseph Frédéric Bonnans and Audrey Hermant. Stability and sensitivity analysis for optimal control problems with a first-order state constraint and application to continuation methods. ESAIM: Control, Optimisation and Calculus of Variations, 14(04):825-863, 2008.

[7] Bernard Bonnard, Ludovic Faubourg, Geneviève Launay, and Emmanuel Trélat. Optimal control with state constraints and the space shuttle re-entry problem. Journal of Dynamical and Control Systems, 9(2):155-199, 2003.

[8] Arthur Earl Bryson and Yu-Chi Ho. Applied optimal control: optimization, estimation, and control. Taylor \& Francis Group, 1975.

[9] Shui-Nee Chow, Wuchen Li, and Haomin Zhou. A newtonlike algorithm for the shortest path based on the method of evolving junctions. Communications in Mathematical Sciences, 1169-1180, 2016.

[10] Shui-Nee Chow, Jun Lu, and Haomin Zhou. Fast numerical methods based on sdes for several problems related to the shortest path. accepted to MAA special issue in honor of Stanley Osher on his 70th birthday.

[11] Shui-Nee Chow, Jun Lu, and Haomin Zhou. Finding the shortest path by evolving junctions on obstacle boundaries (E-JOB): An initial value ODE's approach. Applied and Computational Harmonic Analysis, 2012.

[12] Shui-Nee Chow, Jun Lu, and Haomin Zhou. Shortest path amid 3-d polyhedral obstacles. SIAM scientific computing, 2012 .
[13] Shui-Nee Chow, Tzi-Sheng Yang, and Haomin Zhou. Global optimizations by intermittent diffusion. International Journal of Bifurcation and Chaos, 2013.

[14] John E Dennis and Robert B Schnabel. Numerical methods for unconstrained optimization and nonlinear equations, volume 16. Society for Industrial and Applied Mathematics, 1987.

[15] Roger Fletcher. Practical methods of optimization. John Wiley \& Sons, 2013.

[16] Kikuo Fujimura and Hanan Samet. Planning a time-minimal motion among moving obstacles. Algorithmica, 10(1):41-63, 1993.

[17] Philip E Gill, Walter Murray, and Margaret H Wright. Practical optimization. Academic press, 1981.

[18] Matthew W Harris and Behcet Acikmese. Lossless convexification of non-convex optimal control problems for state constrained linear systems. Automatica, 50(9):23042311,2014

[19] Richard F Hartl, Suresh P Sethi, and Raymond G Vickson. A survey of the maximum principles for optimal control problems with state constraints. SIAM review, 37(2):181218, 1995.

[20] Yunho Hong. Optimal control problems on stratified domains. $\mathrm{PhD}$ thesis, The Pennsylvania State University, 2007.

[21] Elton P Hsu. Stochastic analysis on manifolds, volume 38. American Mathematical Soc., 2002.

[22] Eugene Khmelnitsky. A combinatorial, graph-based solution method for a class of continuous-time optimal control problems.

Mathematics of Operations Research, volume 27(2), pages 312-325, 2002.

[23] Aleksandr Davidovich Loffe, Vladimir Mikhauilovich Tikhomirov, and Karol Makowski. Theory of extremal problems, volume 6. Elsevier, 2009.

[24] Wuchen Li. A study of stochastic differential equations and Fokker-Planck equations with applications. Phd thesis, Georgia Institute of Technology, 2016.

[25] Qun Lin, Ryan Loxton, and Kok Lay Teo. The control parameterization method for nonlinear optimal control: a survey. Journal of Industrial and Management Optimization, pages 275-309, 2014.

[26] Y Liu, S Ito, Hwj Lee, and Kl Teo. Semi-infinite programming approach to continuously-constrained linearquadratic optimal control problems. Journal of Optimization Theory and Applications, 108(3):617-632, 2001.

[27] Eli Livne, David Mineau, Ae Bryson, and Wf Denham. Optimal programming problems with inequality constraints. ii-solution by steepest-ascent. AIAA Journal, 2(1):25-34, 1964.

[28] Ryan Loxton, Qun Lin, Volker Rehbock, and Kok Lay Teo. Control parameterization for optimal control problems with continuous inequality constraints: New convergence results. Numerical Algebra, Control and Optimization, 2(3):571-599, 2012.

[29] Ryan C Loxton, Kok Lay Teo, and Volker Rehbock. Optimal control problems with multiple characteristic time points in the objective and constraints. Automatica, 44(11):2923-2929, 2008.

[30] Ryan C Loxton, Kok Lay Teo, Volker Rehbock, and Ka Fai Cedric Yiu. Optimal control problems with a continuous inequality constraint on the state and the control. Automatica, 45(10):2250-2257, 2009. 
[31] Jun Lu, Yancy Diaz-Mercado, Magnus Egerstedt, Haomin Zhou, and Shui-Nee Chow. Shortest paths through 3-dimensional cluttered environments. accepted to International Conference on robotics and automation, 2014.

[32] K Malanowski. Stability and sensitivity of solutions to nonlinear optimal control problems. Applied Mathematics and Optimization, 32(2):111-141, 1995.

[33] Stephen R Mc Reynolds and Arthur E Bryson Jr. A successive sweep method for solving optimal programming problems. Technical report, DTIC Document, 1965.

[34] Volker Rehbock. Multilevel Optimization of Optimal Control Problems. Optimization Methods and Applications, 125-138, 2001.

[35] Carmeliza Navasca and Arthur J Krener. Patchy solutions of hamilton-jacobi-bellman partial differential equations. In Modeling, estimation and control, pages 251-270. Springer, 2007.

[36] Jorge Nocedal and Stephen J Wright. Numerical optimization. Springer Science+ Business Media, 2006.

[37] Lev Semenovich Pontriagin. The mathematical theory of optimal processes, volume 4. CRC Press, 1962.

[38] Jason L Speyer, Raman K Mehra, and Arthur E Bryson Jr. The separate computation of arcs for optimal flight paths with state variable inequality constraints. Advanced Problems and Methods for space flight optimization, pages 53-68, 1969.

[39] Jur van den Berg and Mark Overmars. Planning timeminimal safe paths amidst unpredictably moving obstacles. The International Journal of Robotics Research, 2008.

[40] Vorgelegt von Andreas Potschka. Handling Path Constraints in a Direct Multiple Shooting Method for Optimal Control Problems. PhD thesis, Ruprecht-Karls-Universitat Heidelberg, 2006. 